OPEN ACCESS

Edited by:

Huanzhong Liu,

Anhui Medical University, China

Reviewed by:

Wei Yan,

Peking University Sixth Hospital,

China

Giovanni Mansueto,

University of Florence, Italy

*Correspondence:

Ahmed Sami Hammam hammamiahmedsami@gmail.com

${ }^{\dagger}$ These authors have contributed equally to this work and share first authorship

Specialty section:

This article was submitted to

Health Psychology,

a section of the journal

Frontiers in Psychology

Received: 05 August 2021 Accepted: 15 November 2021 Published: 14 December 2021

Citation:

Hammami AS, Jellazi $M$, Mahjoub L, Fedhila M and Ouali S (2021) Psychological Impact of the COVID-19 Pandemic on Healthcare

Professionals in Tunisia: Risk and Protective Factors.

Front. Psychol. 12:754047. doi: 10.3389/fpsyg.2021.754047

\section{Psychological Impact of the COVID-19 Pandemic on Healthcare Professionals in Tunisia: Risk and Protective Factors}

\author{
Ahmed Sami Hammami ${ }^{1,2 * t}$, Mohamed Jellazi't, Lobna Mahjoub ${ }^{1}$, Maya Fedhila $^{3}$ and \\ Sonia Ouali ${ }^{1,2}$ \\ ${ }^{1}$ Department of Internal Medicine, CHU F Bourguiba Monastir, Monastir, Tunisia, ${ }^{2}$ Biochemistry Laboratory, LR12ES05 \\ LR-NAFS "Nutrition - Functional Food and Vascular Health", Faculty of Medicine, University of Monastir, Monastir, Tunisia, \\ ${ }^{3}$ Faculty of Dental Medicine of Monastir, Monastir, Tunisia
}

Background: Our study aimed to evaluate the magnitude of different psychological outcomes among Tunisian healthcare professionals (HCPs) during the first wave of the coronavirus disease 2019 (COVID-19) pandemic, and to identify the associated factors.

Methods: Healthcare professionals completed a cross-sectional questionnaire during a 3-week period in the first wave of the COVID-19 pandemic in Tunisia. The survey collected demographic information, factors that may interfere with the psychological outcomes, behavioral changes, and mental health measures. Mental health was assessed using three scales: the Seven-Item Insomnia Severity Index, the TwoItem Patient Health Questionnaire, and the Two-Item Generalized Anxiety Disorder instrument. Multivariable logistic regression was conducted to identify factors associated with psychological outcomes.

Results: A total of 503 HCPs successfully completed the survey, and 493 agreed to enroll in the study: 411 (83.4\%) physicians, 323 (64.2\%) women, and 271 (55\%) with a second-line work position. A significant proportion of HCPs had anxiety (35.7\%), depression (35.1\%), and insomnia (23.7\%). Women, those with a psychiatric history, and those using public transportation had higher proportions for overall symptoms compared with other groups, for example, depression in $44.9 \%$ of female participants vs. $18.2 \%$ of male participants $(p=0.00)$. Those with a previous medical history and nurses had more anxiety and insomnia compared with other groups, for example, anxiety in $45.1 \%$ of nurses, $36.1 \%$ of interns/residents, and $27.5 \%$ of attending physicians $(p=0.04)$. Multivariable logistic regression showed that female gender was a risk factor for all psychological outcomes, whereas psychiatric history was a risk factor for both anxiety and insomnia [odds ratio $(\mathrm{OR})=2.86,95 \% \mathrm{Cl} 1.78-4.60, p=0.00$ for insomnia]. Using protective equipment was associated with a lower risk for depression $(\mathrm{OR}=0.41,95 \% \mathrm{Cl} 0.27-0.62, p=0.00)$ and anxiety. Physical activity was also protective against depression and anxiety $(\mathrm{OR}=0.41,95 \% \mathrm{Cl} 0.25-0.67, p=0.00)$. 
Conclusion: Psychological symptoms are usually overlooked or dismissed by HCPs, although the COVID-19 pandemic played a major role in exacerbating this burden. Prompt psychological support should be endorsed and simple measures, such as physical activity and ensuring the availability of personal protective equipment, are paramount to improve mental health outcomes and the quality of care provided to patients.

Keywords: psychological symptoms, mental health, healthcare professionals, COVID-19 pandemic, risk factors, protective factors

\section{INTRODUCTION}

At the end of December 2019, a novel coronavirus was reported to be the cause of a myriad of pneumonia cases in the Chinese city of Wuhan. The responsible agent was a virus called severe acute respiratory syndrome coronavirus 2 (SARS-CoV-2). In this article, we will refer to the associated disease as the coronavirus disease 2019 (COVID-19) (Lai et al., 2020).

This rapidly spreading infection resulted in an epidemic throughout the entire region and subsequently the entire country of China, followed by a surging number of cases in other countries around the world, which progressively overwhelmed healthcare systems worldwide (Armocida et al., 2020; Xie et al., 2020). By the beginning of March 2020, it was identified as a pandemic (WHO, 2019). Consequently, the WHO highlighted the excessively high burden on healthcare professionals (HCPs), and called for an intervention to address the immediate needs and prevent serious impacts on both physical and mental health in this particular population (Covid 19 Public Health Emergency of International Concern (PHEIC), 2020).

Tunisia was not an exception to the spread of the pandemic, with the first case recorded on March 02, 2020 (Corona virus cases in Tunisia, 2020). As in other affected countries, psychological distress began to propagate, and quickly expanded among HCPs who are directly or indirectly involved in the diagnosis, treatment, and care of patients with COVID-19, and are therefore at high risk of infection (Xiao et al., 2020). Tunisia faced a specific challenge in preparing for the emerging pandemic: a context of political and economic fragility with a precarious financial situation following the democratic transition resulted in the deterioration of the healthcare system and decreasing motivation among HCPs (Zedini et al., 2016; Weilandt, 2018). Among the factors reported to contribute to the mental health burden of these healthcare workers are the overwhelming spread of the life-threatening disease, the gradual increase in numbers of newly diagnosed and suspected cases, the surge in mortality rates, the fear of becoming infected and transmitting the infection to loved ones, the enormous workload, the potential lack of personal protective equipment (PPE), the depletion of certain drugs, the lack of an effective treatment, and

Abbreviations: HCPs, healthcare professionals; COVID-19, novel coronavirus disease; SARS-CoV-2, severe acute respiratory syndrome coronavirus 2; PPE, personnel protective equipment; ISI, Insomnia Severity Index; PHQ-2, Two-Item Patient Health Questionnaire; GAD-2, Two-Item Generalized Anxiety Disorder instrument; MERS-CoV, Middle East respiratory syndrome coronavirus. feelings of being inadequately supported (Muller et al., 2020). Accordingly, it has been reported that during previous viral outbreaks, HCPs endured a high degree of both physical (Xiao et al., 2020) and mental stress during and even years after the end of epidemics (Lancee et al., 2008). The current pandemic has had several psychiatric consequences among healthcare practitioners who treat patients with confirmed or suspected COVID-19 infection (Xiang et al., 2020). Clearly, HCPs should be regarded as a high-risk population because of their higher anxiety, depression, and insomnia levels compared with the general population (Vindegaard and Benros, 2020). In addition, occupational stress in HCPs may exacerbate previously diagnosed psychological problems, and can lead to long-term psychological consequences; most importantly, it may adversely affect the quality of healthcare provided to patients.

A large number of studies in many different countries describe the prevalence of mental health sequelae associated with the COVID-19 pandemic (Brailovskaia et al., 2021). Those research have documented the magnitude of the psychological burden among healthcare workers (Wei et al., 2020; Busch et al., 2021), and have identified risk and protective factors (Wei et al., 2020; Brailovskaia et al., 2021). For instance, an Italian crosssectional survey-based study with a retrospective assessment showed that the COVID-19 outbreak led to life changes in Italian healthcare workers in terms of increase in negative mood, worry, loneliness, fatigue, restlessness, and decrease in happiness (Mansueto et al., 2021). However, none of those studies provided data about the impact of this pandemic on mental health in the Maghreb region. In Tunisia, maintaining health workers' motivation is particularly challenging against a background of low levels of trust in the health system and the government, and this is also the case in other areas of the Middle East and North Africa (MENA) region where governments are perceived to be corrupt. Therefore, estimating the prevalence of risk factors in this population is important to guide public health measures intended to protect health professionals and their families, and to maintain a functioning healthcare system.

Addressing the needs of frontline HCPs during the COVID19 pandemic is an important priority (Remuzzi and Remuzzi, 2020). Accordingly, the aims of our study were to screen for depression, anxiety, and insomnia among Tunisian HCPs during the first wave, and to detect potential risk factors associated with these psychological outcomes. Our findings will, we hope, help to maintain a sustainable, robust healthcare response to 
the pandemic while safeguarding the wellbeing of HCPs in our country and elsewhere.

\section{MATERIALS AND METHODS}

We conducted an online cross-sectional survey at a crucial time when the first wave of the COVID-19 pandemic was sweeping North Africa and Tunisia.

The eligibility criteria were employment as an HCP at any type of public or private health institution in all 24 Tunisian governorates that were registered with the Ministry of Health, and consent for data collection and analysis.

To reach a large number of HCPs, we released information about our survey through the official mailing list of the medical and paramedical professional network in Tunisia, and through health professional groups on Facebook. This approach allowed us to reach large numbers of survey respondents quickly and continuously in all governorates.

All participants were required to provide their consent electronically before responding to the questionnaire. Those who agreed were directed to the questionnaire, and those who declined automatically exited the survey.

Participants were required to respond to all the questions to submit their survey.

All 24 Tunisian governorates were involved, and the responses were then reassembled into three main subgroups according to the Tunisian health department, depending on the prevalence rate in each governorate, i.e., low-, moderate-, or high-prevalence regions (Corona virus cases in Tunisia, 2020).

This study received approval from the research ethics committee of the Faculty of Medicine of Monastir, Tunisia, before the survey was conducted.

The questionnaire was an Internet-based survey containing four sections: basic demographic information, factors that may interfere with psychological outcomes, psychological impact questions, and psychological screening tests.

\section{Demographics}

Data were collected for age (years), gender (male or female), marital status (married or unmarried), medical and psychological history, type of hospital (primary or secondary care center, or tertiary care hospital), technical title (intern/resident, attending/primary care physician, or nurse), and prevalence at the work location (low, moderate, or high).

\section{Factors That May Interfere With Psychological Outcomes}

These factors include work position (frontline for those who work on wards with COVID-19-positive patients and those who staff fever clinic nightshifts, second line for those working in other departments), working in the fever clinic (yes or no), type of transportation used to travel to work (public or private), having children (yes or no), living with family (yes or no), physical activity (yes or no), and the availability of PPE (yes or no).

Fever clinics are facilities usually established outside an emergency department to perform triage and classify patients according to their likelihood of having COVID-19 infection. These clinics have the authority to hospitalize high-risk patients as an interim solution until their subsequent assignment to a specialized unit.

Physical activity in the form of moderate aerobic exercise or more vigorous activity was considered activity performed two or more days per week and lasting at least $40 \mathrm{~min}$ per session. The type of physical activity was not specified in this survey.

\section{Psychological Impact Questions}

These items inquired about experiencing nightmares related to the virus, and about noticeable changes in how respondents performed regular physical examinations for patients.

\section{Psychological Screening Tests}

We used three tests to screen for potential mental disorders among HCPs: the Seven-Item Insomnia Severity Index (ISI), the Two-Item Patient Health Questionnaire (PHQ-2), and the Two-Item Generalized Anxiety Disorder instrument (GAD-2).

The ISI is a concise self-reporting tool that measures the respondent's perception of insomnia. Scores range from 0 to 28 , with $0-7$ indicating no significant insomnia, 8-14 indicating subthreshold insomnia, 15-21 indicating moderate insomnia, and 22-28 indicating severe insomnia (Morin et al., 2011).

We defined clinically significant insomnia as a score $\geq 15$ (merging the latter two categories), and non-clinically significant insomnia as a score $<15$ (merging the first two categories).

Depression and anxiety were assessed with the PHQ-2 and the GAD-2 instrument, respectively. A score of three points was the preferred cut-off to positively screen for depression (Löwe et al., 2010) and anxiety (Kroenke et al., 2007). We divided participants into 2 groups: those who screened positively for depression and anxiety (score $\geq 3$ ) and those whose score was $<3$.

\section{Statistical Analysis}

All data were analyzed with SPSS statistical software, version 26.0 (IBM Corp, Monastir, Tunisia). The significance level was set at $p \leq 0.05$, and all tests were two-tailed. The ranked data were calculated from the counts of each level of symptoms of anxiety, depression, and insomnia in accordance with the pre-established scales we used, and all descriptive data are expressed as numbers $(n)$ and frequencies (\%). Quantitative variables were compared between two groups with the $t$-test, and between more than two groups with analysis of variance. Categorical variables were compared with Fisher's exact test. Univariate analysis was used to evaluate candidate predictors of mental health disorders, and variables with $p$ values $<0.2$ were selected for the multivariable analysis. The associations between outcomes and risk factors are presented as odds ratios (OR) and 95\% CI, after adjustment for confounders.

\section{RESULTS}

The initial total number of participants was 503 (100\%), of whom $10(2 \%)$ declined to participate in the survey. The final number of participants was 493. 
TABLE 1 | Demographic and occupational characteristics of participants.

\begin{tabular}{|c|c|c|c|c|c|c|c|c|c|}
\hline \multirow{3}{*}{ Characteristics } & \multicolumn{9}{|l|}{ No. (\%) } \\
\hline & \multirow[b]{2}{*}{ Total } & \multicolumn{3}{|c|}{ Workplace (COVID risk) } & \multicolumn{2}{|c|}{ Occupation } & \multicolumn{3}{|c|}{ Technical title (3 subgroups) } \\
\hline & & Low & Moderate & High & Physician & Nurse & Intern/Resident & Attending & Nurse \\
\hline Overall & $493(100)$ & $96(19.5)$ & $234(47.5)$ & $163(33.1)$ & $411(83.4)$ & $82(16.6)$ & $302(61.3)$ & $109(22.1)$ & $82(16.6)$ \\
\hline \multicolumn{10}{|l|}{ Gender } \\
\hline Male & $170(33.8)$ & $38(39.6)$ & $74(31.6)$ & $58(35.6)$ & $145(35.3)$ & $25(30.5)$ & $97(32.1)$ & $48(44)$ & $25(30.5)$ \\
\hline Female & $323(64.2)$ & $58(60.4)$ & $160(68.4)$ & $105(64.4)$ & $266(64.7)$ & $57(69.5)$ & 205 (67.9) & $61(56)$ & $57(69.5)$ \\
\hline \multicolumn{10}{|l|}{ Age (years) } \\
\hline$<30$ & $330(66.9)$ & $58(60.4)$ & 165 (70.5) & $107(65.6)$ & $284(69.1)$ & $46(56.1)$ & $265(87.7)$ & $19(17.4)$ & $46(56.1)$ \\
\hline$\geq 30$ & 163 (33.1) & 38 (39.6) & 69 (29.5) & $56(34.4)$ & 127 (30.9) & $36(43.9)$ & $37(12.3)$ & $90(82.6)$ & $36(43.9)$ \\
\hline \multicolumn{10}{|l|}{ Marital status } \\
\hline Unmarried & 351 (69.8) & $65(67.7)$ & $168(71.8)$ & $118(72.4)$ & $299(72.7)$ & $52(63.4)$ & 252 (83.4) & $47(43.1)$ & $52(63.4)$ \\
\hline Married & $142(28.2)$ & $31(32.3)$ & $66(28.2)$ & $45(27.6)$ & $112(27.3)$ & 30 (36.6) & $50(16.6)$ & $62(56.9)$ & $30(36.6)$ \\
\hline Pregnant & $5(1)$ & $2(2.1)$ & $1(0.4)$ & $2(1.2)$ & $5(1.2)$ & $0(0)$ & $3(1)$ & $2(1.8)$ & $0(0)$ \\
\hline \multicolumn{10}{|l|}{ Children } \\
\hline Yes & $113(22.5)$ & $25(26)$ & $52(22.2)$ & $26(22.1)$ & $86(20.9)$ & 27 (32.9) & $24(7.9)$ & $62(56.9)$ & 27 (32.9) \\
\hline No & 380 (75.5) & $71(74)$ & 182 (77.8) & $127(77.9)$ & 325 (79.1) & $55(67.1)$ & $278(92.1)$ & $47(43.1)$ & $55(67.1)$ \\
\hline \multicolumn{10}{|l|}{ Living with family } \\
\hline No & $202(41)$ & $37(38.5)$ & $95(40.6)$ & $70(42.9)$ & $186(45.3)$ & $16(19.5)$ & $160(53)$ & $26(23.9)$ & $16(19.5)$ \\
\hline Yes & $291(59)$ & $59(61.5)$ & 139 (59.4) & $93(57.1)$ & $225(54.7)$ & $66(80.5)$ & $142(47)$ & $83(76.1)$ & $66(80.5)$ \\
\hline \multicolumn{10}{|l|}{ Medical history } \\
\hline Yes & $106(21.5)$ & $22(22.9)$ & $49(20.9)$ & $35(21.5)$ & $94(22.9)$ & $12(14.6)$ & $58(19.2)$ & $36(33)$ & $12(14.6)$ \\
\hline No & 387 (78.5) & $74(77.1)$ & 185 (79.1) & $128(78.5)$ & $317(77.1)$ & $70(85.4)$ & $244(80.8)$ & $73(67)$ & $70(85.4)$ \\
\hline \multicolumn{10}{|l|}{ Psychiatric history } \\
\hline Yes & $63(12.8)$ & $12(12.5)$ & $34(14.5)$ & $17(10.4)$ & $54(13.1)$ & $9(11)$ & 45 (14.9) & $9(8.3)$ & $9(11)$ \\
\hline No & 430 (87.2) & $84(87.5)$ & $200(85.5)$ & $146(89.6)$ & 357 (86.9) & 73 (89) & $257(85.1)$ & $100(91.7)$ & $73(89)$ \\
\hline \multicolumn{10}{|l|}{ Work position } \\
\hline Frontline & $222(45)$ & $47(49)$ & $111(47.4)$ & $64(39.3)$ & $176(42.8)$ & $46(56.1)$ & $140(46.4)$ & $36(33)$ & $46(56.1)$ \\
\hline Second line & $271(55)$ & $49(51)$ & $123(52.6)$ & $99(60.7)$ & $235(57.2)$ & $36(43.9)$ & $162(53.6)$ & $73(67)$ & $36(43.9)$ \\
\hline \multicolumn{10}{|l|}{ Type of hospital } \\
\hline Primary and secondary care center & $130(26.4)$ & $31(32.3)$ & $62(26.5)$ & $37(22.7)$ & $90(21.9)$ & $40(48.8)$ & $23(7.6)$ & $67(61.5)$ & $40(48.8)$ \\
\hline Tertiary care center & 363 (73.6) & $65(67.7)$ & $47.4(73.5)$ & $126(77.3)$ & $321(78.1)$ & $42(51.2)$ & 279 (92.4) & $42(38.5)$ & $42(51.2)$ \\
\hline
\end{tabular}

\section{Demographic Characteristics}

The characteristics of the participants are presented in Table 1.

The final number of participants enrolled in this study was 493 , of whom $411(83.4 \%)$ were physicians and 82 (16.6\%) were nurses. The majority of respondents $(47.5 \%)$ worked in moderate-prevalence regions. Most participants were women $(323,64.2 \%)$, were younger than 30 years old $(330,66.9 \%)$, were unmarried $(351,69.8 \%)$, and worked in tertiary care centers (363, $73.6 \%)$. In most participants, there was no history of medical $(387,78.5 \%)$ or psychiatric illness $(430,87.2 \%)$, and a majority worked as second-line HCPs (271, 55\%).

\section{Factors Associated With Psychological Findings}

A significant proportion of HCPs had depression (35.1\%), anxiety (35.7\%), and insomnia (23.7\%); overall, at least one of these psychological outcomes was present in $45.8 \%$ of HCPs. Women, respondents who had a history of psychiatric illness, and HCPs who used public transportation had higher overall frequencies for all symptoms. Anxiety was noted by $44.9 \%$ of women vs. $18.2 \%$ of men, and insomnia was noted by $29.1 \%$ of women vs. $13.5 \%$ of men (both $p=0.00$ ). Among participants with a history of psychiatric illness, anxiety was noted by $63.5 \%$ vs. $31.6 \%$ of those with no such history $(p=0.00)$, and depression was noted by $54.0 \%$ of the former vs. $32.3 \%$ of the latter $(p=0.01)$. Among those who used public transportation, anxiety was present in $50.4 \%$ vs. $31.2 \%$ of participants who used private transportation $(p=0.00)$ (Table 2). Among HCPs who were $\geq 30$ years old, symptoms of anxiety were more frequent $(73.0 \%)$ compared with younger participants $(60.0 \%, p=0.00)$.

A previous history of medical illness and being employed as a nurse were both associated with a higher frequency of anxiety and insomnia compared with employment as an intern or resident, or as an attending physician. Anxiety was found in $45.1 \%$ of nurses vs. $36.1 \%$ of interns/residents and $27.5 \%$ of attending physicians $(p=0.04)$, and insomnia was seen in $23.7 \%$ of nurses, $19.9 \%$ of interns/residents, and $21.1 \%$ of attending physicians $(p=0.00)$. In addition, participants with a history of medical illness showed more anxiety and insomnia compared with those with no such 
TABLE 2 | Anxiety, depression, and insomnia measures in the total cohort and subgroups.

\begin{tabular}{|c|c|c|c|c|c|c|c|c|c|}
\hline \multirow[t]{2}{*}{ Variables } & \multicolumn{3}{|c|}{ PHQ-2, depression } & \multicolumn{3}{|c|}{ GAD-2, anxiety } & \multicolumn{3}{|c|}{ ISI, insomnia } \\
\hline & Depressed & Not depressed & $p$ value & Anxiety & No anxiety & $p$ value & Insomnia & No insomnia & $p$ value \\
\hline Total & $173(35.1)$ & 320 (64.9) & & $176(35.7)$ & $317(64.3)$ & & $117(23.7)$ & 367 (76.3) & \\
\hline \multicolumn{10}{|l|}{ Age (years) } \\
\hline$<30$ & $122(37)$ & $208(63)$ & 0.214 & $198(60)$ & $132(40)$ & $<0.01$ & $74(22.4)$ & $256(77.6)$ & 0.331 \\
\hline$\geq 30$ & $51(31.3)$ & $112(68.7)$ & & $119(73)$ & $44(27)$ & & $43(26.4)$ & $120(73.6)$ & \\
\hline \multicolumn{10}{|l|}{ Gender } \\
\hline Male & $42(24.7)$ & $128(75.3)$ & $<0.01$ & $31(18.2)$ & $139(81.8)$ & $<0.01$ & $23(13.5)$ & $147(86.5)$ & $<0.01$ \\
\hline Female & $131(40.6)$ & $192(59.4)$ & & 145 (44.9) & $178(55.1)$ & & $94(29.1)$ & $229(70.9)$ & \\
\hline \multicolumn{10}{|l|}{ Medical history } \\
\hline Yes & $44(41.5)$ & $62(58.5)$ & 0.118 & $48(45.3)$ & $58(54.7)$ & 0.02 & $34(32.1)$ & $72(67.9)$ & 0.023 \\
\hline No & 129 (33.3) & $258(66.7)$ & & $128(33.1)$ & 259 (66.9) & & $83(21.4)$ & 304 (78.6) & \\
\hline \multicolumn{10}{|l|}{ Psychiatric history } \\
\hline Yes & $34(54)$ & $29(46)$ & $<0.01$ & $40(63.5)$ & $23(36.5)$ & $<0.01$ & $33(52.4)$ & $30(47.6)$ & $<0.01$ \\
\hline No & 139 (32.3) & $291(67.7)$ & & 136 (31.6) & $294(68.4)$ & & $84(19.5)$ & $346(80.5)$ & \\
\hline \multicolumn{10}{|l|}{ Work position } \\
\hline Frontline & 88 (39.6) & $134(60.4)$ & 0.055 & $76(34.2)$ & $146(65.8)$ & 0.539 & $62(27.9)$ & $160(72.1)$ & 0.047 \\
\hline Second line & 85 (31.4) & $186(68.6)$ & & $100(36.9)$ & $171(63.1)$ & & $55(20.3)$ & $216(79.7)$ & \\
\hline \multicolumn{10}{|l|}{ Fever clinic } \\
\hline Yes & $65(41.1)$ & 93 (58.9) & 0.034 & $97(61.4)$ & $61(38.6)$ & 0.204 & $46(29.1)$ & $112(70.9)$ & 0.036 \\
\hline No & $108(32.2)$ & $227(67.8)$ & & $220(65.7)$ & $115(34.3)$ & & $71(21.2)$ & $264(78.8)$ & \\
\hline \multicolumn{10}{|l|}{ Technical title } \\
\hline Interns and residents & $103(34.1)$ & $199(65.9)$ & 0.161 & $109(36.1)$ & $193(63.9)$ & 0.042 & $60(19.9)$ & $242(80.1)$ & $<0.01$ \\
\hline Attending and primary care physicians & $34(31.2)$ & $75(68.8)$ & & $30(27.5)$ & $79(72.5)$ & & $23(21.1)$ & $86(78.9)$ & \\
\hline Nurses & $36(43.9)$ & $46(56.1)$ & & $37(45.1)$ & $45(54.9)$ & & $34(23.7)$ & 48 (76.3) & \\
\hline \multicolumn{10}{|l|}{ Protective equipment } \\
\hline Yes & $71(26.4)$ & 198 (73.6) & $<0.01$ & $80(29.7)$ & $189(70.3)$ & $<0.01$ & $53(19.7)$ & $216(80.3)$ & 0.021 \\
\hline No & $102(45.5)$ & $122(54.5)$ & & $96(42.9)$ & $128(57.1)$ & & $64(28.6)$ & $160(71.4)$ & \\
\hline \multicolumn{10}{|l|}{ Physical activity } \\
\hline Yes & $39(24.8)$ & $118(75.2)$ & $<0.01$ & $36(22.9)$ & $121(77.1)$ & $<0.01$ & $28(17.8)$ & $129(82.2)$ & 0.035 \\
\hline No & $134(39.9)$ & $202(60.1)$ & & $140(41.7)$ & $196(58.3)$ & & $89(26.5)$ & $247(73.5)$ & \\
\hline \multicolumn{10}{|l|}{ Children } \\
\hline Yes & $36(31.9)$ & $77(68.1)$ & 0.434 & $30(26.5)$ & $83(74.3)$ & 0.021 & $29(25.7)$ & $84(74.3)$ & 0.583 \\
\hline No & $137(36.1)$ & $243(63.9)$ & & $146(38.4)$ & $234(61.6)$ & & $88(23.2)$ & $292(76.8)$ & \\
\hline \multicolumn{10}{|l|}{ Public transportation } \\
\hline Yes & $54(47)$ & $61(53)$ & $<0.01$ & $58(50.4)$ & $57(49.6)$ & $<0.01$ & $35(30.4)$ & $80(69.6)$ & 0.037 \\
\hline No & $119(31.5)$ & $259(68.5)$ & & 118 (31.2) & $260(68.8)$ & & $82(21.7)$ & 296 (78.3) & \\
\hline \multicolumn{10}{|l|}{ Living with family } \\
\hline Yes & $102(59)$ & $71(41)$ & 0.529 & $113(38.83)$ & $178(61.17)$ & 0.049 & $73(25.1)$ & 218 (74.9) & 0.23 \\
\hline No & $189(59.1)$ & 131 (40.9) & & 63 (31.18) & 139 (68.81) & & $44(21.8)$ & 158 (78.2) & \\
\hline
\end{tabular}

history. Among the former, anxiety was seen in $45.3 \%$ vs. $33.1 \%$ of the latter $(p=0.02)$. Insomnia was seen in $32.1 \%$ of respondents with a previous medical history vs. $21.4 \%$ of those with no such history $(p=0.02)$.

Healthcare professionals employed in frontline positions had more frequent insomnia $(27.9 \%)$ than those working in secondline positions $(20.3 \%, p=0.04)$. Individuals working in fever clinics were more prone to have symptoms of depression (41.1\% vs. $32.2 \%, p=0.03)$ and insomnia $(29.1 \%$ vs. $21.2 \%$, $p=0.03)$ compared with those who did not work in this clinical setting. We highlight the finding that $33.7 \%$ of all participants had nightmares related to the virus, and $66.3 \%$ reported behavioral changes in performing routine clinical examinations of their patients.

Compared with those who lived alone, HCPs who lived with their family were more prone to anxiety. Among HCPs living with their family, anxiety was found in $38.8 \%$ vs. $31.18 \%$ of those who lived alone $(p=0.04)$.

Physical activity and the availability of PPE were associated with a lower frequency of psychiatric outcomes. Anxiety was noted in $22.9 \%$ of participants who were physically active vs. $41.7 \%$ of those who were not $(p=0.00)$. Depression was noted in $26.4 \%$ of those who had access to PPE vs. $45.5 \%$ of those who $\operatorname{did} \operatorname{not}(p=0.00)$. 
TABLE 3 | Risk and protective factors for mental health outcomes identified by multivariable regression.

\begin{tabular}{|c|c|c|c|}
\hline Variable & $\begin{array}{l}\text { No. cases/No. } \\
\text { total cases (\%) }\end{array}$ & $\begin{array}{l}\text { Adjusted OR } \\
\qquad(95 \% \mathrm{Cl})\end{array}$ & $p$ value \\
\hline \multicolumn{4}{|l|}{ PHQ-2, depression symptoms } \\
\hline \multicolumn{4}{|l|}{ Gender } \\
\hline Men & $42 / 170(24.7)$ & 1 (reference) & 0.005 \\
\hline Women & 131/323 (40.6) & $1.88(1.21-2.92)$ & \\
\hline \multicolumn{4}{|l|}{ Physical activity } \\
\hline Yes & $39 / 157$ (24.8) & $0.49(0.31-0.78)$ & 0.002 \\
\hline No & 134/336 (39.9) & 1 (reference) & \\
\hline \multicolumn{4}{|l|}{ Protective equipment } \\
\hline Yes & $71 / 269(26.4)$ & $0.41(0.27-0.62)$ & 0.000 \\
\hline No & $102 / 124$ (45.5) & 1 (reference) & \\
\hline \multicolumn{4}{|l|}{ Public transportation } \\
\hline Yes & $54 / 115(47)$ & 1.69 (1.06-2.69) & 0.027 \\
\hline No & $119 / 378(31.5)$ & 1 (reference) & \\
\hline \multicolumn{4}{|l|}{ GAD-2, Anxiety symptoms } \\
\hline \multicolumn{4}{|l|}{ Gender } \\
\hline Men & $31 / 170(18.2)$ & 1 (reference) & 0.000 \\
\hline Women & $145 / 323$ (44.9) & $2.86(1.78-4.60)$ & \\
\hline \multicolumn{4}{|l|}{ Psychiatric history } \\
\hline Yes & 40/63 (63.5) & $3.05(1.66-5.63)$ & 0.000 \\
\hline No & 136/430 (31.6) & 1 (reference) & \\
\hline \multicolumn{4}{|l|}{ Physical activity } \\
\hline Yes & 36/157 (22.9) & $0.41(0.25-0.67)$ & 0.000 \\
\hline No & $140 / 336(41.7)$ & 1 (reference) & \\
\hline \multicolumn{4}{|l|}{ Protective equipment } \\
\hline Yes & $80 / 269(29.7)$ & $0.65(0.43-0.98)$ & 0.044 \\
\hline No & $96 / 224$ (42.9) & 1 (reference) & \\
\hline \multicolumn{4}{|l|}{ Type of hospital } \\
\hline Primary and secondary care center & $53 / 130(40.8)$ & $1.76(1.01-3.08)$ & 0.045 \\
\hline Tertiary care center & 123/363 (33.9) & 1 (reference) & \\
\hline \multicolumn{4}{|l|}{ Living with family } \\
\hline Yes & $113 / 291(64.2)$ & $1.62(1.02-2.60)$ & 0.041 \\
\hline No & 63/202 (35.8) & 1 (reference) & \\
\hline \multicolumn{4}{|l|}{ Public transportation } \\
\hline Yes & $58 / 115(50.4)$ & $1.73(1.06-2.83)$ & 0.029 \\
\hline No & $118 / 378$ (31.2) & 1 (reference) & \\
\hline \multicolumn{4}{|l|}{ ISI, insomnia symptoms } \\
\hline \multicolumn{4}{|l|}{ Gender } \\
\hline Men & 23/170 (13.5) & 1 (reference) & 0.002 \\
\hline Women & $94 / 323(29.1)$ & $2.29(1.34-3.92)$ & \\
\hline \multicolumn{4}{|l|}{ Psychiatric history } \\
\hline Yes & 33/63 (52.4) & $3.82(2.09-6.96)$ & 0.000 \\
\hline No & $84 / 430(19.5)$ & 1 (reference) & \\
\hline
\end{tabular}

Multivariable logistic regression (Table 3), after confounders were controlled, identified the following risk factors for depression: female gender (OR 1.88, 95\% CI 1.21-2.92, $p=0.01$ ) and using public transportation (OR 1.69, 95\% CI 1.06-2.69, $p=0.03)$. As protective factors, this analysis identified physical activity (OR $0.49,95 \%$ CI $0.31-0.78, p=0.00$ ) and availability of appropriate PPE (OR 0.41, 95\% CI $0.27-0.62, p=0.00$ ).

Five risk factors were independently related to anxiety among HCPs: history of psychiatric illness (OR 3.05, 95\% CI 1.66-5.63, $p=0.00$ ), female gender (OR 2.86, 95\% CI 1.78-4.60, $p=0.00$ ), working in primary or secondary care centers (OR 1.76, 95\% CI 1.01-3.08, $p=0.05$ ), using public transportation (OR 1.73, $95 \%$ CI 1.06-2.83, $p=0.03$ ), and living with family (OR 1.62, $95 \%$ CI 1.02-2.60, $p=0.04)$. Conversely, the factors found to be protective against anxiety were physical activity (OR 0.41, 95\% CI $0.25-0.67, p=0.00$ ) and PPE (OR 0.65, 95\% CI 0.43-0.98, $p=0.04)$.

Regarding insomnia, we identified two risk factors. A history of a psychiatric illness showed the strongest association (OR 3.82, 95\% CI 2.09-6.96, $p=0.00$ ), followed by female gender (OR 2.29; $95 \%$ CI $1.34-3.92, p=0.00)$.

\section{DISCUSSION}

This study examined the presence of anxiety, depression, and insomnia symptoms among health workers in Tunisia during the early phase of the COVID-19 pandemic. During the previous decade, Tunisia faced few epidemics without a significant impact on its healthcare system. The 2012 West Nile virus epidemics (Hammami et al., 2017) and the 2013 MERS-COV outbreaks (Abroug et al., 2014) are two examples, but neither of them was comparable to the COVID-19 pandemic in terms of amplitude and consequences on country-wide aspects of healthcare, economy, the agri-food system, and households (Zouhair et al., 2020).

The current pandemic emerged in Tunisia at a time when the country was grappling with an economic crisis, in addition to the political instability (Weilandt, 2018). In fact, Tunisia is facing an unprecedented crisis due to continuous political turmoil and the unfolding economic and financial meltdown, exacerbated by the COVID-19 pandemic.

The pandemic started with a significant delay compared with the European countries that had already reached the exponential phase of infection distribution. However, in the setting of a fragile healthcare system, Tunisian HCPs found themselves unprepared either psychologically or logistically to face this rapidly evolving pandemic (Chaari and Golubnitschaja, 2020), especially at its first onset.

Furthermore, as a lower middle-income country (Classification of countries according to their income, 2019), Tunisia has very limited resources; hence, HCPs struggled with a lack of available tests and with discrepancies in test performance between different regions, which led to a heterogeneous picture of the infection distribution and mortality rates (Chaari and Golubnitschaja, 2020). They also faced difficulties due to insufficient medical equipment and PPE, and limited hospitalization capacity (Fredj and Chérif, 2020). These factors consequently made HCPs susceptible to negative mental health outcomes.

This study is the first of its kind in the Maghreb region. Countries in this region (Algeria, Morocco, Tunisia, and Libya) are characterized by generally similar healthcare systems, thus our sample may reflect, to some extent, the mental health situation among HCPs during this pandemic in other Maghreb countries. 
This Tunisian study characterizes the impact of the pandemic during its early phase on mental health in HPCs, including a wide range of medical professionals from all over the country. Previous studies in different parts of the world were cross-sectional or longitudinal, and included essentially doctors and nurses. A meta-analysis of this earlier research showed that on average, two out of every five HCPs endured negative psychological outcomes during the COVID-19 pandemic, for example, anxiety, depression, insomnia, and other worrisome outcomes (Muller et al., 2020). Another meta-analysis was undertaken on a global scale combined studies of influenza and coronavirus epidemics, and reported substantial proportions of psychological symptoms among HCPs (Serrano-Ripoll et al., 2020). Thus, HCPs, worldwide have been challenged psychologically in the context of the current global health crisis.

As previously highlighted in other research (Lai et al., 2020), our survey documented significant frequencies of psychological symptoms among HCPs during the COVID-19 pandemic: $35.7 \%$ had anxiety, $35.1 \%$ had depression, and $23.7 \%$ had insomnia. Fekih-Romdhane et al. (2020) conducted a cross-sectional study among solely residents $(n=210)$ in Tunisia, and the Depression, Anxiety and Stress Scale (DASS) scores they obtained disclosed severe or extremely severe levels of depression in $30.5 \%$, anxiety in $24.3 \%$, and stress in $18.6 \%$ of their participants. The ISI scores in their study revealed a high prevalence rate of insomnia (41.4\%) (Fekih-Romdhane et al., 2020). Similarly, Dong and Gao (2021) reported a high prevalence of anxiety (24.15\%) and insomnia (39.83\%). These authors used the Self-Reported Anxiety Scale (SAS) and the ISI to screen for anxiety and insomnia, respectively.

In a study by Khanal et al. (2020), health workers in Nepal completed an online questionnaire; the findings disclosed symptoms of anxiety in $41.9 \%$, depression symptoms in $37.5 \%$, and symptoms of insomnia in $33.9 \%$. Anxiety and depression were measured with the 14-item Hospital Anxiety and Depression Scale (HADS: 0-21), and insomnia was measured with the seven-item ISI (range 0-28).

Pappa et al. (2020) in their systematic review and metaanalysis of 13 cross-sectional studies $(n=33,062)$, reported a high prevalence rate of anxiety, depression, and insomnia during the COVID-19 pandemic. On the other hand, other researchers reported much lower rates. In Singapore, only 14.5\% of HCPs screened positive for anxiety, and only $8.9 \%$ had depression. These results may be explained by the fact that new protective equipment was available in Singapore to protect HCPs and help them deal with the COVID-19 calamity (Tan et al., 2020). Differences in prevalence rates between studies can also be explained by the different settings where each study was conducted. Another contributing factor may be the use of different scoring scales.

In the present study, health workers who were women, and those with a history of mental health problems, had a higher odds of exhibiting anxiety, depression, and insomnia symptoms compared with men and to participants with no such history. Similar findings were observed in other studies (Lai et al., 2020; Prasad et al., 2021). The difference between genders may be explained in part by women's greater propensity to express psychological distress compared with men (Zhang and Wing, 2006; McLean et al., 2011; Abate, 2013; Mansueto and Faravelli, 2021). These findings may also reflect the predominance of women in patient-facing roles. Other factors that can affect women healthcare workers' wellbeing include role strain, difficulties maintaining work-life balance, the consequences associated with family members, gender-related discrimination, and a lack of sufficient support systems.

Healthcare professionals with a history of mental illness experienced more anxiety and insomnia, which is consistent with the results of Zhang W.R. et al. (2020). Khanal et al. (2020) conducted a cross-sectional study among 475 health workers who treated patients with COVID-19 in Nepal and noted that a history of medication for mental health problems was significantly associated with a higher likelihood of experiencing symptoms of anxiety, depression, and insomnia compared with colleagues with no such history.

A similar finding was observed in a study by Zhu et al. (2020) who noted that workers with a history of mental health problems were more likely to have anxiety, depression, and stress. A recent study of 1,685 participants in the USA showed that HCPs with a history of psychiatric illness were at a greater risk for significant mental burden (Young et al., 2021). In fact, HCPs with a mental illness are inherently predisposed and more vulnerable to the appearance of psychiatric symptoms because of their mental fragility, and hence their lower threshold for distress.

Healthcare professionals with medical history experienced more anxiety and insomnia which is consistent with the results of Zhang W.R. et al. (2020). We believe that this is due to their assumption of having potentially worse outcomes if they get infected with COVID-19 (Wei et al., 2020; Wolff et al., 2020), especially, during the first wave when there was no previously established information about the COVID-19 sequelae and its interference with other illnesses. Our findings are supported by Zhang et al., who showed that having an organic disease was an independent factor for insomnia, anxiety, and depression.

Based on the multivariable regression model, we found that female gender and psychiatric history were independent risk factors for mental health outcomes in nearly all measures.

In the present study, nurses reported more symptoms of anxiety and insomnia than their colleagues (69.5\% of whom were women). Nurses are considered essential HCPs deeply involved in patient management with close, constant physical proximity (e.g., repeated monitoring and recording signs, drug administration, and blood sampling, etc.) and hence frequently exposed to the highest risk of contagion (Barrett et al., 2020). Similarly, another highly exposed group were those working as frontline HCPs or in fever clinics, who experienced more insomnia than secondline workers. These subgroups are responsible for providing direct care to patients with COVID-19 and for collecting sputum specimens for virus detection.

Interestingly, we found that using public transportation to go to work was a source of worse mental health outcomes in all measures, and it was shown by multivariable regression that public transport was a major risk factor for depression and anxiety. These results are mainly due to the heightened stress level caused by the risk of contagion when in close contact with people who might be infected (Arora et al., 2020). 
Similarly, HCPs who were in close contact with their family members expressed heightened levels of anxiety compared with those who did not live with their families. This finding is attributable to the fear of being COVID-19-positive and the consequent risk of transmitting the virus to their families.

Coping psychologically with a pandemic can be arduous for healthcare workers (Wong et al., 2005). The origin of psychological issues may be related with fear of infection, fear of transmitting the virus to their relatives (we note that residing with family members was an independent risk factor for anxiety in our participants), lack of information about the virus, concerns about the shortage of PPE, absence of psychological support, and the burden of long working hours (Muller et al., 2020). Additionally, one-third of our participants reported experiencing nightmares related to the virus, and this may further indicate the overwhelming affliction endured by healthcare workers during the pandemic, in consonance with similar results published by Herrero et al. (2020). A large proportion of HCPs (66.3\%) also adopted a different attitude toward patients (e.g., eviction or incomplete routine clinical examination), which further highlights their fear of infection owing to the lack of PPE (45.4\% of HCPs reported insufficient PPE). It is important to note that a fearful or suspicious attitude may jeopardize the quality of care provided to all types of patients.

Our findings showed that HCPs who were physically active reported fewer symptoms in all mental health measures. This result parallels findings reported by Maugeri et al. (2020), who noted a significant positive correlation between physical activity and mental wellbeing in the general population during the current pandemic. Other studies support the benefits of physical activities and show that it buffers the negative effects of bad experiences and traumatic events, and can provide resilience in facing challenges (Richards et al., 2015; Brailovskaia and Margraf, 2017). In the current pandemic, physical activity has been shown to be negatively associated with the psychological burden induced by COVID-19: those who engage in a regular exercise routine may have a less stressful pandemic experience, and thus tend to cope better with everyday distress (Brailovskaia et al., 2021).

Our results also indicated that the availability of appropriate PPE was a protective factor against depression and anxiety. This finding is consistent with a study by Zhang S.X. et al. (2020), who showed that having PPE was associated with less distress. Also, Dong and Gao (2021) identified the unavailability of PPE as a significant risk factor for both anxiety and insomnia.

In the light of our findings and as the COVID-19 pandemic continues, providing appropriate strategies will be of great importance in supporting HCPs, especially those who are involved in the treatment of patients with COVID-19. To avoid negative psychological outcomes, efforts should be tailored and directed toward high-risk groups, i.e., female nurses who have a history of psychiatric illness, and HCPs with comorbidities.

For example, female nurses working in frontline positions during the health crisis should be allowed more rest and more days off, their psychological symptoms should be regularly monitored, and the threshold for seeking psychological specialist support should be lowered. In addition, HCPs with a known psychiatric history should be screened for any aggravation of their symptoms, and care should be sought immediately if their symptoms worsen.

The use of public transportation during the pandemic was shown to be an important contributor to the mental burden borne by our participants; thus policymakers should endorse measures that favor social distancing and frequent sanitization of public transportation. If economically feasible, dedicated private transportation should be used by HCPs. Finally, to ensure that optimum healthcare is provided to patients, more advocacy tools should be implemented to persuade decision-makers and healthcare administration officials of the importance of PPE availability, which is known to be a prominent protective factor against the mental burden faced by HCPs.

Our study has several limitations. First, because this was a cross-sectional survey-based study, casual inferences are limited. Second, the possibility of selection bias was non-negligible because all HCPs were invited to participate, and those who participated may have been more aware of their mental health issues than those who did not. Third, the PHQ-2 and GAD-2 psychological scales were used mainly for screening purposes; they do not evaluate the degree or the severity of symptoms. Fourth, we did not screen for any aggravation of symptoms during the pandemic, e.g., preexisting mental health symptoms vs. new symptoms, and this limitation applies particularly to participants with a previous history of psychiatric illness. Moreover, other potential confounding variables including past or current pharmacologic treatment or data on past psychological intervention in HCPs could have impacted the outcome of the present study reflecting the mental health consequences of COVID-19 (Carvalho et al., 2016; Cosci et al., 2016; Swartz, 2020). Additionally, the 3 -week duration of the data collection process may have affected the quality of the responses, given that the incidence of COVID-19 and perceptions related to infection may have differed between the first and last day of the survey.

Despite these limitations, our study provides important baseline information on many psychological morbidities endured by healthcare workers during this critical period. Our findings can serve as an important tool enabling policymakers to provide guidance to HCPs about effective strategies to protect their mental health. Because of the increasingly worrisome mental health outcomes among HCPs, their symptoms may become more severe with time, thus we recommend conducting further research on the long-term psychological implications.

\section{CONCLUSION}

The COVID-19 pandemic has had a deleterious effect on healthcare workers' mental health, with evidence of an increase in depressive, anxiety, and insomnia symptoms negatively impacting the quality of care they provide to patients. Hence, special attention should be paid to HCPs who are female nurses, and to those who have a history of psychiatric illness and are prone to mental disorders. Also, urgent measures 
should be implemented toward more rapid and effective risk communication, endorsement of protective factors that could help to manage this burden, and more efficient identification of those who are in distress.

\section{DATA AVAILABILITY STATEMENT}

The original contributions presented in the study are included in the article/supplementary material, further inquiries can be directed to the corresponding author.

\section{ETHICS STATEMENT}

This study was approved by the Institutional Committee of Fattouma Bourguiba University Hospital in accordance with the Declaration of Helsinki. Electronic informed consent was obtained from the participants. The patients/participants

\section{REFERENCES}

Abate, K. H. (2013). Gender disparity in prevalence of depression among patient population: a systematic review. Ethiop. J. Health Sci. 23, 283-288. doi: 10.4314/ ejhs.v23i3.11

Abroug, F., Slim, A., and Ouanes-Besbes, L. (2014). Family cluster of Middle East respiratory syndrome coronavirus infections, Tunisia, 2013. Emerg. Infect. Dis. 20, 1527-1530. doi: 10.3201/eid2009.140378

Armocida, B., Formenti, B., Ussai, S., Palestra, F., and Missoni, E. (2020). The Italian health system and the COVID-19 challenge. Lancet Public Health 5:e253. doi: 10.1016/S2468-2667(20)30074-8

Arora, A., Jha, A. K., Alat, P., and Das, S. S. (2020). Understanding coronaphobia. Asian J. Psychiatry 54:102384. doi: 10.1016/j.ajp.2020.102384

Barrett, E. S., Horton, D. B., Roy, J., Gennaro, M. L., Brooks, A., Tischfield, J., et al. (2020). Prevalence of SARS-CoV-2 infection in previously undiagnosed health care workers at the onset of the U.S. COVID-19 epidemic. medRxiv doi: 10.1101/2020.04.20.20072470

Brailovskaia, J., Cosci, F., Mansueto, G., Miragall, M., Herrero, R., Baños, R. M., et al. (2021). The association between depression symptoms, psychological burden caused by Covid-19 and physical activity: An investigation in Germany, Italy, Russia, and Spain. Psychiatry Res. 295:113596. doi: 10.1016/j.psychres. 2020.113596

Brailovskaia, J., and Margraf, J. (2017). Facebook Addiction Disorder (FAD) among German students-A longitudinal approach. PLoS One 12:e189719. doi: 10. 1371/journal.pone.0189719

Busch, I. M., Moretti, F., Mazzi, M., Wu, A. W., and Rimondini, M. (2021). What We Have Learned from Two Decades of Epidemics and Pandemics: A Systematic Review and Meta-Analysis of the Psychological Burden of Frontline Healthcare Workers. Psychother. Psychosom. 90, 178-190. doi: 10. $1159 / 000513733$

Carvalho, A. F., Sharma, M. S., Brunoni, A. R., Vieta, E., and Fava, G. A. (2016). The Safety, Tolerability and Risks Associated with the Use of Newer Generation Antidepressant Drugs: A Critical Review of the Literature. Psychother. Psychosom. 85, 270-288. doi: 10.1159/000447034

Chaari, L., and Golubnitschaja, O. (2020). Covid-19 pandemic by the "real-time" monitoring: the Tunisian case and lessons for global epidemics in the context of 3PM strategies. Epma J. 11, 1-6. doi: 10.1007/s13167-020-00207-0

Classification of Countries According to Their Income (2019). Available online at: https://data.worldbank.org/country/tunisia?display=graph $\% 22 \% 3 \mathrm{EBanco}$ (accessed March, 2019).

Corona Virus Cases in Tunisia (2020). Available online at: https://www. worldometers.info/coronavirus/country/tunisia/ (accessed March, 2020). provided their written informed consent to participate in this study.

\section{AUTHOR CONTRIBUTIONS}

$\mathrm{AH}$ and $\mathrm{MJ}$ conceptualized and designed the study and supervised the writing and analyses. LM and MF contributed to data collection and analysis. SO critically reviewed the manuscript. All authors approved the final manuscript as submitted.

\section{ACKNOWLEDGMENTS}

We thank Abdulrazzak Abyad, Coordinator of the Middle-East Primary Care Research Network, for his kind advice in the field, and K. Shashok (AuthorAID in the Eastern Mediterranean) for editing the use of English in the manuscript.

Cosci, F., Mansueto, G., Faccini, M., Casari, R., and Lugoboni, F. (2016). Sociodemographic and clinical characteristics of benzodiazepine long-term users: Results from a tertiary care center. Compr. Psychiatry 69, 211-215. doi: 10.1016/ j.comppsych.2016.06.008

Covid 19 Public Health Emergency of International Concern [PHEIC] (2020). Global Research and Innovation Forum: Towards a Research Roadmap. Available online at: https://covid19-evidence.paho.org/handle/20.500.12663/ 714 (accessed January, 2020).

Dong, H. S., and Gao, J. J. (2021). Prevalence of insomnia and anxiety among healthcare workers during the COVID-19 pandemic in Jilin Province. Braz. J. Med. Biol. Res. 54:e10602. doi: 10.1590/1414-431X2020e10602

Fekih-Romdhane, F., Snene, H., Jebri, A., Ben Rhouma, M., and Cheour, M. (2020). Psychological impact of the Pandemic COVID-19 Outbreak Among Medical Residents in Tunisia. Asian J. Psychiatr. 53:102349. doi: 10.1016/j.ajp.2020. 102349

Fredj, H. B., and Chérif, F. (2020). Novel Corona virus disease infection in Tunisia: Mathematical model and the impact of the quarantine strategy. Chaos Solitons Fractals 138:109969. doi: 10.1016/j.chaos.2020.109969

Hammami, S., Hassine, T. B., Conte, A., Amdouni, J., De Massis, F., Sghaier, S., et al. (2017). West Nile disease in Tunisia: an overview of 60 years. Vet. Ital. 53, 225-234. doi: 10.12834/VetIt.1181.6565.2

Herrero, S. M. A., Parra, S. J., Diaz, C. T., Arias, A. E. M., Muñoz, M. J., Alvarez, D. Y. M. J., et al. (2020). Sleep characteristics in health workers exposed to the COVID-19 pandemic. Sleep Med. 75, 388-394. doi: 10.1016/j.sleep.2020.08.013

Khanal, P., Devkota, N., Dahal, M., Paudel, K., and Joshi, D. (2020). Mental health impacts among health workers during COVID-19 in a low resource setting: a cross-sectional survey from Nepal. Global Health 16:89. doi: 10.1186/s12992020-00621-z

Kroenke, K., Spitzer, R. L., Williams, J. B., Monahan, P. O., and Löwe, B. (2007). Anxiety disorders in primary care: prevalence, impairment, comorbidity, and detection. Ann. Intern. Med. 146, 317-325. doi: 10.7326/0003-4819-146-5200703060-00004

Lai, J., Ma, S., Wang, Y., Cai, Z., Hu, J., Wei, N., et al. (2020). Factors Associated With Mental Health Outcomes Among Health Care Workers Exposed to Coronavirus Disease 2019. JAMA Netw. Open 3:e203976. doi: 10. 1001/jamanetworkopen.2020.3976

Lancee, W. J., Maunder, R. G., and Goldbloom, D. S. (2008). Prevalence of psychiatric disorders among Toronto hospital workers one to two years after the SARS outbreak. Psychiatr. Serv. 59, 91-95. doi: 10.1176/ps.2008.5 9.1.91

Löwe, B., Wahl, I., Rose, M., Spitzer, C., Glaesmer, H., Wingenfeld, K., et al. (2010). A 4-item measure of depression and anxiety: validation and standardization of 
the Patient Health Questionnaire-4 (PHQ-4) in the general population. J. Affect Disord. 122, 86-95. doi: 10.1016/j.jad.2009.06.019

Mansueto, G., and Faravelli, C. (2021). Stressful life events and psychosis gender differences. Stress Health 2021:3067. doi: 10.1002/smi.3067

Mansueto, G., Lopes, F. L., Grassi, L., and Cosci, F. (2021). Impact of COVID-19 outbreak on Italian healthcare workers versus general population: Results from an online survey. Clin. Psychol. Psychother. 2021:13. doi: 10.1002/cpp.2644

Maugeri, G., Castrogiovanni, P., Battaglia, G., Pippi, R., D’Agata, V., Palma, A., et al. (2020). The impact of physical activity on psychological health during Covid-19 pandemic in Italy. Heliyon 6:e04315. doi: 10.1016/j.heliyon.2020. e04315

McLean, C. P., Asnaani, A., Litz, B. T., and Hofmann, S. G. (2011). Gender differences in anxiety disorders: prevalence, course of illness, comorbidity and burden of illness. J. Psychiatr. Res. 45, 1027-1035. doi: 10.1016/j.jpsychires.2011. 03.006

Morin, C. M., Belleville, G., Bélanger, L., and Ivers, H. (2011). The Insomnia Severity Index: psychometric indicators to detect insomnia cases and evaluate treatment response. Sleep 34, 601-608. doi: 10.1093/sleep/34.5.601

Muller, A. E., Hafstad, E. V., Himmels, J. P. W., Smedslund, G., Flottorp, S., Stensland, S., et al. (2020). The mental health impact of the covid-19 pandemic on healthcare workers, and interventions to help them: A rapid systematic review. Psychiatry Res. 293:113441. doi: 10.1016/j.psychres.2020.113441

Pappa, S., Ntella, V., Giannakas, T., Giannakoulis, V. G., Papoutsi, E., and Katsaounou, P. (2020). Prevalence of depression, anxiety, and insomnia among healthcare workers during the COVID-19 pandemic: A systematic review and meta-analysis. Brain Behav. Immun. 88, 901-907.

Prasad, K., McLoughlin, C., Stillman, M., Poplau, S., Goelz, E., Taylor, S., et al. (2021). Prevalence and correlates of stress and burnout among U.S. healthcare workers during the COVID-19 pandemic: A national cross-sectional survey study. EClin. Med. 35:100879. doi: 10.1016/j.eclinm.2021.100879

Remuzzi, A., and Remuzzi, G. (2020). COVID-19 and Italy: what next? Lancet 395, 1225-1228.

Richards, J., Jiang, X., Kelly, P., Chau, J., Bauman, A., and Ding, D. (2015). Don't worry, be happy: Cross-sectional associations between physical activity and happiness in 15 European countries. BMC Public Health 15:53. doi: 10.1186/ s12889-015-1391-4

Serrano-Ripoll, M. J., Meneses-Echavez, J. F., Ricci-Cabello, I., Fraile-Navarro, D., Fiol-deRoque, M. A., Pastor-Moreno, G., et al. (2020). Impact of viral epidemic outbreaks on mental health of healthcare workers: a rapid systematic review and meta-analysis. J. Affect Disord 277, 347-357. doi: 10.1016/j.jad.2020.08.034

Swartz, H. A. (2020). The Role of Psychotherapy During the COVID-19 Pandemic. Am J Psychother 73, 41-42. doi: 10.1176/appi.psychotherapy.20200015

Tan, Z., Khoo, D. W. S., Zeng, L. A., Tien, J. C., Lee, A. K. Y., Ong, Y. Y., et al. (2020). Protecting health care workers in the front line: Innovation in COVID-19 pandemic. J. Glob. Health 10:010357. doi: 10.7189/jogh.10.010357

Vindegaard, N., and Benros, M. E. (2020). COVID-19 pandemic and mental health consequences: Systematic review of the current evidence. Brain Behav. Immun. 89, 531-542. doi: 10.1016/j.bbi.2020.05.048

Wei, Y. Y., Wang, R. R., Zhang, D. W., Tu, Y. H., Chen, C. S., Ji, S., et al. (2020). Risk factors for severe COVID-19: Evidence from 167 hospitalized patients in Anhui, China. J. Infect. 81, e89-e92. doi: 10.1016/j.jinf.2020.04.010

Weilandt, R. (2018). Socio-economic challenges to Tunisia's democratic transition. Eur. View 17, 210-217. doi: 10.1177/1781685818805681

WHO (2019). Timeline: WHO's COVID-19 response. Geneva: WHO.

Wolff, D., Nee, S., Hickey, N. S., and Marschollek, M. (2020). Risk factors for Covid19 severity and fatality: a structured literature review. Infection 2020, 1-14. doi: 10.1007/s15010-020-01509-1
Wong, T. W., Yau, J. K., Chan, C. L., Kwong, R. S., Ho, S. M., Lau, C. C., et al. (2005). The psychological impact of severe acute respiratory syndrome outbreak on healthcare workers in emergency departments and how they cope. Eur. J. Emerg. Med. 12, 13-18. doi: 10.1097/00063110-200502000-00005

Xiang, Y. T., Jin, Y., and Cheung, T. (2020). Joint International Collaboration to Combat Mental Health Challenges During the Coronavirus Disease 2019 Pandemic. JAMA Psychiatry 77, 989-990. doi: 10.1001/jamapsychiatry.2020. 1057

Xiao, J., Fang, M., Chen, Q., and He, B. (2020). SARS, MERS and COVID-19 among healthcare workers: A narrative review. J. Infect. Public Health 13, 843-848. doi: 10.1016/j.jiph.2020.05.019

Xie, J., Tong, Z., Guan, X., Du, B., and Qiu, H. (2020). Critical care crisis and some recommendations during the COVID-19 epidemic in China. Intensive Care Med. 46, 837-840. doi: 10.1007/s00134-020-05979-7

Young, K. P., Kolcz, D. L., O’Sullivan, D. M., Ferrand, J., Fried, J., and Robinson, K. (2021). Health Care Workers' Mental Health and Quality of Life During COVID-19: Results From a Mid-Pandemic, National Survey. Psychiatr. Serv. 72, 122-128. doi: 10.1176/appi.ps.202000424

Zedini, C., Ben Cheikh, A., Limam, M., Henrichi, Y., Mellouli, M., El Ghardallou, M., et al. (2016). Determinants of Paramedical Staff Motivation in a Tunisian Hospital. Sante Publique 28, 613-622.

Zhang, B., and Wing, Y. K. (2006). Sex differences in insomnia: a meta-analysis. Sleep 29, 85-93. doi: 10.1093/sleep/29.1.85

Zhang, S. X., Liu, J., Afshar Jahanshahi, A., Nawaser, K., Yousefi, A., Li, J., et al. (2020). At the height of the storm: Healthcare staff's health conditions and job satisfaction and their associated predictors during the epidemic peak of COVID-19. Brain Behav. Immun. 87, 144-146. doi: 10.1016/j.bbi.2020.05.010

Zhang, W. R., Wang, K., Yin, L., Zhao, W. F., Xue, Q., Peng, M., et al. (2020). Mental Health and Psychosocial Problems of Medical Health Workers during the COVID-19 Epidemic in China. Psychother. Psychosom. 89, 242-250. doi: $10.1159 / 000507639$

Zhu, Z., Xu, S., Wang, H., Liu, Z., Wu, J., Li, G., et al. (2020). COVID-19 in Wuhan: Sociodemographic characteristics and hospital support measures associated with the immediate psychological impact on healthcare workers. EClinicalMedicine 24:100443. doi: 10.1016/j.eclinm.2020.100443

Zouhair, E. D. E., Aymen, F., Thouraya, L., Manfred, W., and Breisinger, A. C. (2020). The Impact of COVID-19 on Tunisia's Economy, Agri-food System, and Households. Tunisian Institute of Competitiveness and Quantitative Studies. Washington, D.C: Intl Food Policy Res Inst.

Conflict of Interest: The authors declare that the research was conducted in the absence of any commercial or financial relationships that could be construed as a potential conflict of interest.

Publisher's Note: All claims expressed in this article are solely those of the authors and do not necessarily represent those of their affiliated organizations, or those of the publisher, the editors and the reviewers. Any product that may be evaluated in this article, or claim that may be made by its manufacturer, is not guaranteed or endorsed by the publisher.

Copyright (c) 2021 Hammami, Jellazi, Mahjoub, Fedhila and Ouali. This is an openaccess article distributed under the terms of the Creative Commons Attribution License (CC BY). The use, distribution or reproduction in other forums is permitted, provided the original author(s) and the copyright owner(s) are credited and that the original publication in this journal is cited, in accordance with accepted academic practice. No use, distribution or reproduction is permitted which does not comply with these terms. 